\title{
Optimization of Drilling Path Using the Bees Algorithm
}

Shafie Kamaruddin (0000-0002-1108-3046), Mohamad Naqiuddin Rosdi (0000-0002-3223- 2223), Nor Aiman Sukindar (0000-0002-3223-2223)

Department of Manufacturing and Materials Engineering, Faculty of Engineering, International Islamic University Malaysia (IIUM), Kuala Lumpur, Malaysia.E-mail: shafie@iium.edu.my

Optimization is the process of finding the best possible solutions to a problem. It has been widely used in various areas especially in engineering problems. One of the common issues that is faced by some of the manufacturers is finding drilling sequences of multiple holes. By drilling multiple holes with the least total path length, the manufacturer can save a lot of time and it can increase the productivity of the company. Thus, this study focuses on the drilling path of the multiple holes problem which has been solved by other researchers. This study uses the Bees Algorithm to find the best sequence of drilling holes (minimum total path length) and the results found are compared with the result of other algorithms. In addition to results comparison with other algorithms, the results obtained are verified with simulation results using MasterCAM software. The results comparison shows that the Bees Algorithm achieved comparable performance compared to other algorithms.

Keywords: Bees Algorithm, Optimization, Drilling

\section{Introduction}

One of the most common machining processes in the industries is hole drilling process. The main issue in this process is point to point tool movement during the drilling process. Normally, the drilling process is using Computer Numerical Control (CNC) machine, particularly for Printed Circuit Board (PCB) application $[1,2]$. This process is time consuming when involves multiples holes as to move the tool bit from one point to another increases the machining time. Thus, optimization of the drilling path could reduce the machining time which will also improve the overall productivity in the industries [3]. For this reason, optimization plays an important role in addressing the issues of finding the drilling path from all the possible paths available. The definition of optimization is the process of finding the most effective or best solution or condition [4].

The most common method to solve optimization problem are using Sequential Quadratic Programming (SQP), Recursive Quadratic Programming, Sequential Linearization Algorithm and Evolutionary Programming [5,6,7]. However, with rapid development of computer processing capability, a new subset of Artificial Intelligence named Swarm Intelligence (SI) has been developed [8]. Examples of SI are Ant Colony Optimization (ACO) algorithm, Particle Swarm Optimization (PSO) algorithm, and the Bees Algorithm.

One of the well-known benchmark optimization problems is Travelling Salesman Problem (TSP) [1]. This problem arises when a salesman needs to find the shortest route to travel from several cities. Thus, instead of randomly travel to any cities to make sales, applying optimization method helps salesman to find route with the least total distance. This TSP problem is similar to the problem that will be discussed further in this paper which is drilling path optimization using the Bees Algorithm [9].

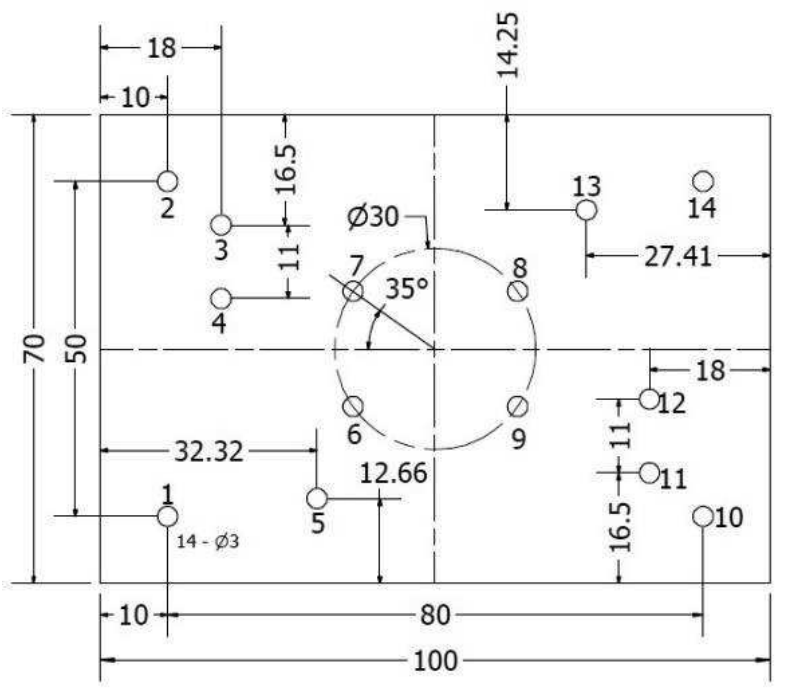

Fig. 1 Image of the 14-holes benchmark problem

In this paper, the problems that would be further discussed is the CNC Drilling on the multiple holes of the Printed Circuit Board (PCB). The holes drilling problem used in this study has been applied by other algorithms such are Bat Algorithm (BA), Simulated Kalman Filter (SKF) and Particle Swarm Optimization (PSO) algorithm [10,11,12,13,14]. This problem 
was chosen because it is well known benchmark problem and widely used to compare the performance of optimization algorithms. The drilling problem has 14holes PCB with the same sizes and different distances between the two holes. Fig. 1 shows the drawing of the 14 holes PCB showing the position of the holes.

The Bees Algorithm is a combination of both local search and global search using exploitation and exploration strategies. The flowchart of the basic Bees Algorithm is shown in Fig. 2 [15]. The algorithm begins with initialisation where (ns) scout bees being sent to search space randomly. The fitness values of each site are evaluated via objective function and ranked according to fitness values. Then, the local search stage of the Bees Algorithm begins with selecting (m) best sites out of (ns) scout bees. The selection of these sites is based on fitness values. These best sites are divided into two subgroups which are elite sites (ne) and nonelite best sites (nb-ne). The local search process starts with sending recruited bees randomly within the neighbourhood of the best sites. More recruit bees are sent to elite sites compared to the best sites [16]. The next stage is global search is where the unselected (nsne) bees are sent randomly across the search space. The last step is merging the fittest of each elite site, the fittest of each best site, and unselected bees. Then, it is sorted according to the fitness value and the process continues until the global optimum is found [17].

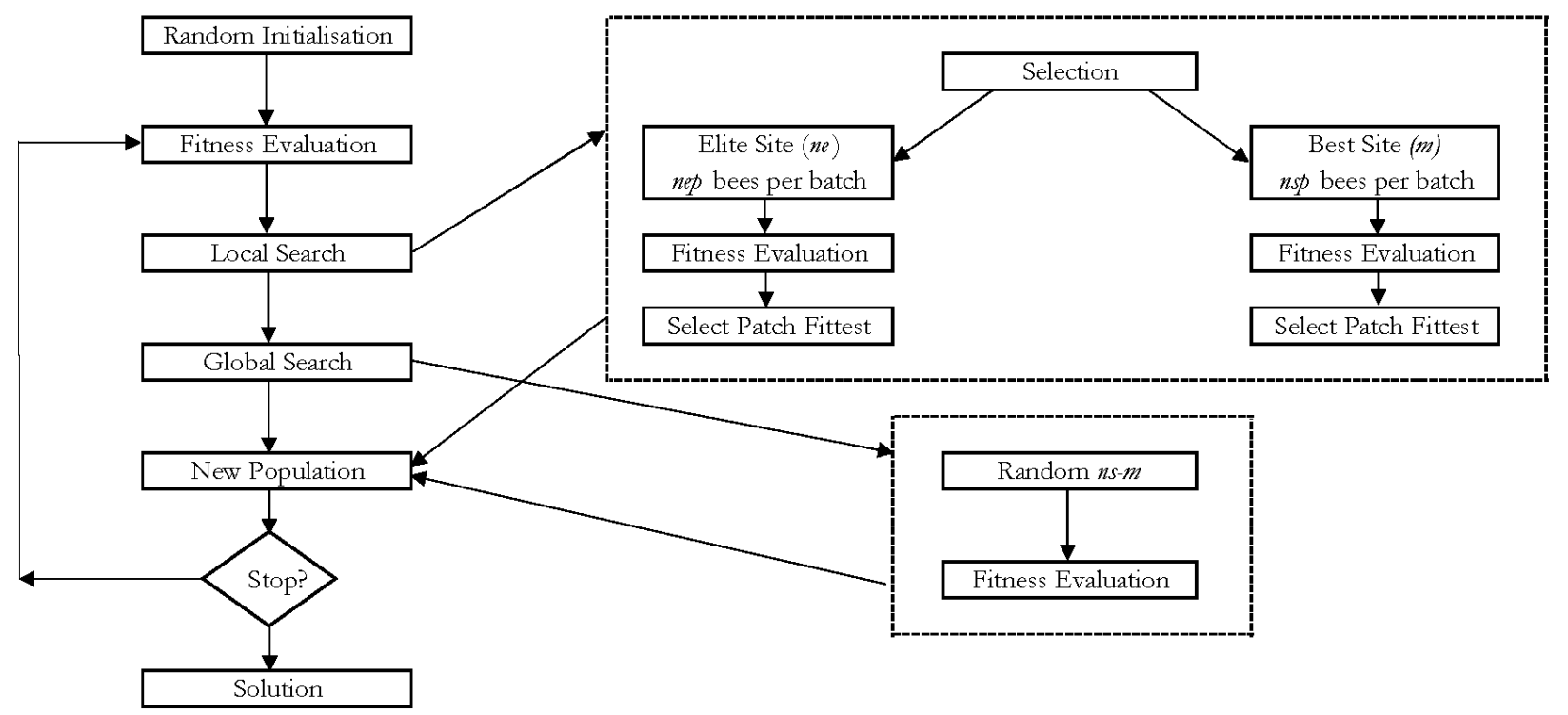

Fig. 2 Flowchart of the Bees Algorithm

\section{Methodology}

Similarly with other algorithms, parameter tuning is required to find the best solution. The Bees algorithm requires several parameters to be set manually before running the algorithm. Each set of parameters consisting of the number of scout bees in the selected sites (ns), number of best sites in the selected sites (m), number of elite sites in the selected best sites (ne), number of recruited bees in the elite sites (nep), number of recruited bees in the non-elite best sites (nsp) and the remaining unselected scout bees (ns-m). The value used to initialize the parameters used in the Bees Algorithm is important as it might affect the results. A rule of thumb can be used as a guide to select a feasible value of parameters for the Bees Algorithm. The first rule is the number of bees selected (nep and nsp) must be less than or equal to the number of initial scout bees (ns) but greater than 0 . Then, the number of recruited bees in elite sites (nep) must exceed the number of recruiters for selected best sites (nsp). Lastly, the number of selected best sites (m) should be larger than the number of elite sites (ne) [15]. Tab. 1 shows the 10 sets of parameters that were established manually to be used for solving problem in this study.

Tab. 1 Combination of parameters used

\begin{tabular}{|l|l|l|l|l|l|}
\hline Set & ns & ne & nep & m & nsp \\
\hline 1 & 10 & 4 & 10 & 9 & 1 \\
\hline 2 & 10 & 6 & 20 & 9 & 10 \\
\hline 3 & 10 & 7 & 20 & 9 & 10 \\
\hline 4 & 10 & 2 & 10 & 6 & 5 \\
\hline 5 & 10 & 2 & 10 & 5 & 5 \\
\hline 6 & 10 & 3 & 10 & 6 & 5 \\
\hline 7 & 10 & 7 & 30 & 9 & 15 \\
\hline 8 & 12 & 5 & 30 & 9 & 15 \\
\hline 9 & 10 & 4 & 10 & 8 & 5 \\
\hline 10 & 20 & 7 & 30 & 9 & 15 \\
\hline
\end{tabular}

In this study, $R$ software was used to run the Bees Algorithm. It is a programming language used for data manipulation, statistical analysis, and data visualization. It has the ability to manipulate, calculate and display data [18]. In addition, MasterCAM software was also used this study to verify the results of the Bees 
Algorithm. The best drilling sequence drilling found by the Bees Algorithm is simulated using Master CAM software. It is an effective tool to generate, execute and simulate tool paths. This software also commonly used in the manufacturing industries. There are many features available this software such as cutting time, air time, tool changing time and others. However, the focus on this study is to verify the total length path only.

\section{Results and Discussion}

This section describes the results of application of

Tab. 2 Path length found at different parameters

\begin{tabular}{|l|l|l|l|l|}
\hline Set & Best $(\mathrm{mm})$ & Worst $(\mathrm{mm})$ & Mean $(\mathrm{mm})$ & $\begin{array}{l}\text { Standard } \\
(\mathrm{mm})\end{array}$ \\
\hline 1 & 280.00 & 370.66 & 328.19 & 20.54 \\
\hline 2 & 280.00 & 374.50 & 334.83 & 22.26 \\
\hline 3 & 280.00 & 376.32 & 337.24 & 22.29 \\
\hline 4 & 280.00 & 388.84 & 339.11 & 27.87 \\
\hline 5 & 280.00 & 390.68 & 345.05 & 26.10 \\
\hline 6 & 280.00 & 391.16 & 330.85 & 25.83 \\
\hline 7 & 280.00 & 393.44 & 346.10 & 23.18 \\
\hline 8 & 292.30 & 392.88 & 343.74 & 23.35 \\
\hline 9 & 295.20 & 407.20 & 350.10 & 26.76 \\
\hline 10 & 303.60 & 403.68 & 356.71 & 26.87 \\
\hline
\end{tabular}

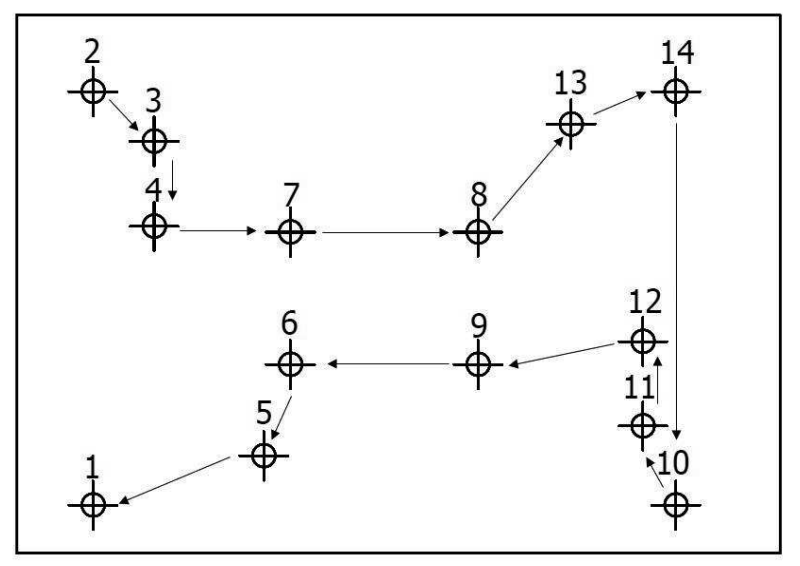

Fig. 3 The best sequence of the toolpath

Comparison of the search progress of best sequence and worst sequence found by the Bees Algorithm is shown in Figure 5. This figure describes the Bees Algorithm takes about 49 iterations to find the best sequence (1-5-6-9-12-11-10-14-13-8-7-4-3-2) with total path length of $280 \mathrm{~mm}$. Meanwhile for the worst sequence (1-5-9-14-10-11-12-6-7-4-3-2-13-8), the path length found is $303.60 \mathrm{~mm}$ at 49 iterations. In general, finding the least fitness value at a smaller number of iterations shows it ability to find solution at faster rate. Thus, this shows the best sequence has faster convergence rate compared to worst sequence.

Tab. 3 shows comparison of result obtained by the the Bees Algorithm on tool path optimization problem. Tab. 2 shows the results of the ten sets of the parameters based on the best, worst, mean and the standard deviation over 50 runs for each set of parameters found by the Bees Algorithm.

It is observed that, the Bees Algorithm found similar best solutions in most of the set of parameters. This result also shows that the worst result is using parameter set 10 . Other set of parameters (8 and 9) found solution close to $280 \mathrm{~mm}$. The sequence of the best tool path sequence and worst tool path sequences are illustrated in Fig. 3 and Fig. 4, respectively.

Bees Algorithm using parameter set 1 with other algorithms based on the best path length, mean path length and standard deviation $[10,11,12]$. Based on the results comparison shown in Tab. 3, the minimum path length found by the Bees Algorithm is $280 \mathrm{~mm}$, which is similar with most of other algorithms. However, the Bat Algorithm achieved slightly lower length path, which is about $276.76 \mathrm{~mm}$. Then, there are other algorithms that neither achieve $280 \mathrm{~mm}$ which are GA and PSO algorithm. This shows the superiority of the Bees Algorithm over other algorithms in finding best solution for this problem.

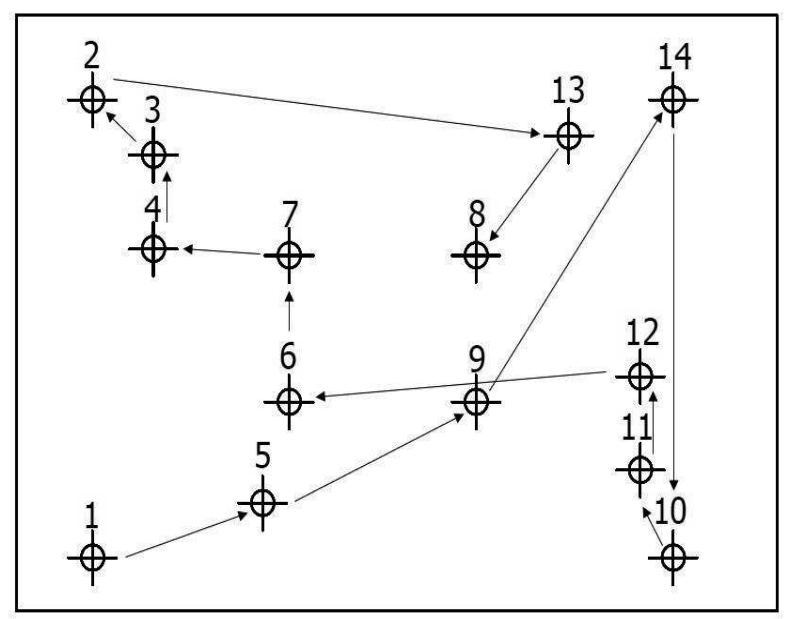

Fig. 4 The worst sequence of the problem 


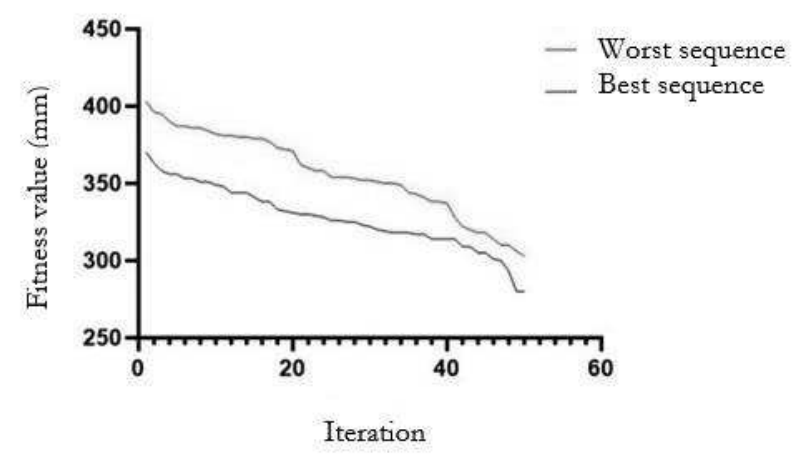

Fig. 5 Search progress comparison

Furthermore, based on the results obtained in this study, other comparison criteria such as the mean, standard deviation and number of iterations are also considered. The performance of the algorithms is compared. Based on results comparison in Tab. 3 in term of best path length over 50 runs, Bat Algorithm found the best path length among those algorithms which is $276.76 \mathrm{~mm}$. Meanwhile, the Bees Algorithm and SKF found the best tool path length of $280 \mathrm{~mm}$. In term of mean path length, the Bat Algorithm obtained the best result which is $276.82 \mathrm{~mm}$. The Bees Algorithm obtained the worst result which is $328.19 \mathrm{~mm}$. In term of the standard deviation, the Bat Algorithm shows the best value which is $0.663 \mathrm{~mm}$ compared to $20.54 \mathrm{~mm}$ of the Bees Algorithm. Despite the Bat Algorithm found better tool path length than the Bees Algorithm, the Bees Algorithm still found better tool path length compared to PSO algorithm. This result shows that the Bees Algorithm has good performance on this optimization problem.

In addition, based on the results found the Bees Algorithm, the sequences are also verified using the Master CAM software. The sequences found the Bees Algorithm are simulated to verify the tool path. This simulation verified the sequences found the Bees Algorithm. The first step is to create the $2 \mathrm{D}$ rectangle of 14 holes as used by other algorithms which also known as 14-hole benchmark problems using Auto$C A D$. This software is selected because it is easier to be used than the other software in term of drafting and designing. Besides, the Master CAM can import any files that being save in the dwg.file which is one of the format on the AutoCAD.

Then, the dwg.file is imported into the Master $C A M$ software. Next, the Mill process is selected followed by selecting the drill option. After that, the sequence of the holes was selected. For the settings, the drilling parameters are set to be $0 \mathrm{~mm}$. It is because the main objective of using Master CAM in this paper is on the tool path. The best sequence found by the Bees Algorithm, the Bat Algorithm and SKF are simulated on Master $C A M$. The simulation results using Master $C A M$ verified and showed similar with result found by the Bees Algorithm run using the $R$ software. However, the best sequence of the Bat Algorithm simulated using Master $C A M$ found different total path length. For SKF best sequence, the total path length is verified as the total path length similar as reported.

Tab. 3 Results of comparison with other algorithms

\begin{tabular}{|l|l|l|l|l|}
\hline & $\begin{array}{l}\text { The } \\
\text { Bees } \\
\text { Algorithm }\end{array}$ & $\begin{array}{l}\text { Bat } \\
\text { Algorithm }\end{array}$ & $\begin{array}{l}\text { Simulated Kalman } \\
\text { Filter (SKF) }\end{array}$ & $\begin{array}{l}\text { Particle Swarm Opti- } \\
\text { mization (PSO) Algo- } \\
\text { rithm }\end{array}$ \\
\hline Best path length (mm) & 280.00 & 276.76 & 280.00 & 297.99 \\
\hline $\begin{array}{l}\text { Mean path length } \\
\text { (mm) }\end{array}$ & 328.19 & 276.82 & - & 298.18 \\
\hline $\begin{array}{l}\text { Standard Deviation of } \\
\text { path length (mm) }\end{array}$ & 20.54 & 0.663 & - & 2.129 \\
\hline Iterations & 49 & Below 50 & 27 & 93 \\
\hline
\end{tabular}

\section{Conclusion}

In this paper, a study of finding an optimized drilling path using the Bees Algorithm has been presented. The Bees Algorithm is applied on the drilling path problem with the objective to find to minimize total path length. Several set of Bees Algorithm parameters were used to find the best sequence. This study has shown that the best path length found by the Bees Algorithm is similar with SKF which is $280 \mathrm{~mm}$. Furthermore, comparison with other algorithms shows that the Bees Algorithm found better path length compared to PSO algorithm. Taken together, these results suggest that the Bees Algorithm has the capability to find better or at least same solution compared to other algorithms. Despite showing good performance, the scope of this study was limited for this specific 14 holes benchmark problem only. Thus, future works could include applying more complex problems which involves drilling higher number of holes.

\section{Acknowledgement}

This study was supported by FRGS grant No. FRGS/1/2019/TK03/UIAM/02/3 from Ministry of Education Malaysia (MOE). Authors also grateful to the International Islamic University of Malaysia (IIUM) which made this study possible. 


\section{References}

[1] LIM, W.C.E., KANAGARAJ, G. \& PONNAMBALAM, S.G. (2016). A hybrid cuckoo search-genetic algorithm for hole-making sequence optimization. Journal of Intelligent Manufacturing, 27, pp. 417-429.

[2] ACIU, R. \& CIOCARLIE, H. (2014). G-code optimization algorithm and its application on Printed Circuit Board drilling. 2014 IEEE 9th IEEE International Symposium on Applied Computational Intelligence and Informatics (SACI), pp. 4347.

[3] LIM, W.C.E., KANAGARAJ, G. \& PONNAMBALAM, S.G. (2014). PCB Drill Path Optimization by Combinatorial Cuckoo Search Algorithm. The Scientific World Journal, vol. 2014, Article ID 264518, 11 pages.

[4] JAMIL, M., YANG, X. S., \& ZEPERNICK, H. J. D. (2013). Test Functions for Global Optimization: A Comprehensive Survey. In Swarm Intelligence and Bio-Inspired Computation (pp. 193222).

[5] KANTOR, M., CHALUPA, M., SOUČEK, J., BÍLKOVÁ, E. \& NOWAK, P. (2020). Application of genetic algorithm methods for water turbine blade shape optimization. Manufacturing Technology, 20, pp. 453-458.

[6] ZHANG, W., HOU, L., GAN, Y., XU, C., BU, X. \& LIN, H. (2019). Parallel Optimization of the Balancing and Sequencing for Mixed-model Assembly Lines. Manufacturing Technology, 19, pp. 537-544.

[7] MARTOWIBOWO, S.Y. \& KEMALA DAMANIK, B. (2021). Optimization of Material Removal Rate and Surface Roughness of AISI 316L under Dry Turning Process using Genetic Algorithm. Manufacturing Technology, 21, pp. 373-380.

[8] KAR, A. K. (2016). Bio Inspired Computing A Review of Algorithms and Scope of Applications. Expert Systems with Applications, 59, pp. $20-32$.

[9] OYSU, C., \& BINGUL, Z. (2007). Tool path optimization using genetic algorithms. In Proceedings of the 2007 International Conference on Genetic and Evolutionary Methods, GEM (pp. 120-126).
[10] DIYALEY, S., BURMAN BISWAS, A., \& CHAKRABORTY, S. (2019). Determination of the optimal drill path sequence using bat algorithm and analysis of its optimization performance. Journal of Industrial and Production Engineering, 36(2), pp. 97-112.

[11] ZHU, G. Y. (2006). Drilling path optimization based on swarm intelligent algorithm. 2006 IEEE International Conference on Robotics and Biomimetics, ROBIO 2006, 1, pp. 193-196.

[12] ABDUL AZIZ, N. H., AB AZIZ, N. A., IBRAHIM, Z., RAZALI, S., ABAS, K. H., \& MOHAMAD, M. S. (2016). A Kalman Filter approach to PCB drill path optimization problem, 2016 IEEE Conference on Systems, Process and Control (ICSPC), pp. 33-36.

[13] DAADOO M., ELEYAN D., TARAPIAH S., ATALLA S., ELEYAN A. (2018). Computer Numerical Control-PCB Drilling Machine with Efficient Path Planning - Case Study 2. Aut. Control Comp. Sci. 52, pp. 451-463.

[14] ISMAIL M. M., OTHMAN M.A., SULAIMAN H. A., MISRAN M. H., RAMLEE R. H., ABIDIN A. F. NORDIN Z., N. A., ZAKARIA M. I., AYOB M. N., YAKOP F. (2012). Firefly algorithm for path optimization in PCB holes drilling process. 2012 International Conference on Green and Ubiquitous Technology, pp. 110-113.

[15] PHAM, D. T., \& CASTELLANI, M. (2009). The bees algorithm: Modelling foraging behaviour to solve continuous optimization problems. Proceedings of the Institution of Mechanical Engineers, Part C: Journal of Mechanical Engineering Science, 223(12), pp. 2919-2938.

[16] PHAM, D.T., CASTELLANI, M. (2014). Benchmarking and comparison of nature-inspired population-based continuous optimisation algorithms. Soft Computing, 18, pp. 871-903.

[17] KAMARUDDIN, S., BAHARI, M. S., PHAM, D. T., HAMZAS, M. F. M. A., ZAKARIA, S. (2019). Bees algorithm enhanced with Nelder and Mead method for numerical function optimisation. AIP Conference Proceedings. 1, 2129.

[18] R CORE TEAM (2020). R: A language and environment for statistical computing. $\mathrm{R}$ Foundation for Statistical Computing, Vienna, Austria. URL https://www.R-project.org/. 